\title{
Upscaled modeling in multiphase flow applications
}

\author{
V. GINTING, R. EWING, Y. EFENDIEV and R. LAZAROV \\ Department of Mathematics and Institute for Scientific Computation \\ Texas A \& M University, College Station, TX 77843-3404 \\ E-mails: ginting@math.tamu.edu / richard-ewing@tamu.edu / \\ efendiev@math.tamu.edu / lazarov@math.tamu.edu
}

\begin{abstract}
In this paper we consider upscaling of multiphase flow in porous media. We propose numerical techniques for upscaling of pressure and saturation equations. Extensions and applications of these approaches are considered in this paper. Numerical examples are presented.
\end{abstract}

Mathematical subject classification: $65 \mathrm{~N} 99$.

Key words: multiscale, macro-diffusion, heterogeneous.

\section{Introduction}

The modeling of multiphase flow in porous formations is important for both environmental remediation and the management of petroleum reservoirs. Practical situations involving multiphase flow include the dispersal of a non-aqueous phase liquid in an aquifer or the displacement of a non-aqueous phase liquid by water. In the subsurface, these processes are complicated by the effects of permeability heterogeneity on the flow and transport. Simulation models, if they are to provide realistic predictions, must accurately account for these effects. However, because permeability heterogeneity occurs at many different length scales, numerical flow models cannot in general resolve all of the variation of scales. Therefore, approaches are needed for representing the effects of subgrid scale variations on larger scale flow results.

On the fine (fully resolved) scale, the subsurface flow and transport of $N$ components can be described in terms of an elliptic (for incompressible systems) pressure equation coupled to a sequence of $N-1$ hyperbolic (in the absence \#576/03. Received: 14/IV/03. Accepted: 14/VIII/03. 
of dispersive and capillary pressure effects) conservation laws. In this paper we address the upscaling of both pressure and saturation equations.

Traditional approaches for scale up of pressure equations generally involve the calculation of effective media properties. In these approaches the fine scale information is built into the effective media parameters, and then the problem on the coarse scale is solved. We refer to $[5,10,19,20]$ for more discussion on upscaled modeling in multiphase flows. Recently, a number of approaches have been proposed in which the methods have the ability to capture the small scales effect on the large scale solution without directly resolving the small scale details $[1,13,23,24]$. In particular, a number of approaches have been introduced where the coupling of small scale information is performed through a numerical formulation of the global problem by incorporating the fine features of the problem into base elements. In this work we develop a similar approach using finite volume framework. Because of their conservative feature, finite volume methods are often preferred in applications such as flow in porous media. Our methodology is similar to multiscale finite element methods [18, 23]. We discuss numerical implementation as well as some applications of our approach.

Though there are a number of technical issues associated with subgrid models for the pressure equation, the lack of robustness of existing coarse scale models is largely due to the treatment of the hyperbolic transport equations. Previous approaches for the coarse scale modeling of transport in heterogeneous oil reservoirs include the use of pseudo relative permeabilities $[3,7,31,32]$, the application of nonuniform or flow-based coarse grids [12], and the use of volume averaging and higher moments $[10,16,17]$. Our methodology for subgrid upscaling of the hyperbolic (or convection dominated) equations uses volume averaging techniques. In particular, we employ perturbation analysis to derive the macro-diffusion that represents the effects of subgrid heterogeneities. Numerical computation of macro-diffusion is addressed in this paper. The macro-diffusion, in particular, can be written as a covariance between the velocity fluctuations and fine scale quantity that represents the length of fine scale trajectories. For the computation of fine scale quantities, we use detailed information that is contained in multiscale base functions. Further application of our subgrid upscaling approaches to other porous media flow problems such as multiphase flow in unsaturated/saturated media (Richards' equation) is discussed. Numerical example 
is presented. The theoretical considerations of the approaches are brief and will be presented elsewhere.

The paper is organized as follows. In the next section we discuss the main upscaling procedures that will be used, and section 3 is devoted to the numerical results.

\section{Fine and coarse scale models}

We consider two phase flow in a reservoir $\Omega$ under the assumption that the displacement is dominated by viscous effects; i.e., we neglect the effects of gravity, compressibility, and capillary pressure. Porosity will be considered to be constant. The two phases will be referred to as water and oil, designated by subscripts $w$ and $o$, respectively. We write Darcy's law, with all quantities dimensionless, for each phase as follows:

$$
\mathbf{v}_{j}=-\frac{k_{r j}(S)}{\mu_{j}} \mathbf{k} \cdot \nabla p,
$$

where $\mathbf{v}_{j}$ is the phase velocity, $\mathbf{k}$ is the permeability tensor, $k_{r j}$ is the relative permeability to phase $j(j=o, w), \mu_{j}$ is its corresponding viscosity, $S$ is the water saturation (volume fraction), and $p$ is pressure. In this work, a single set of relative permeability curves is used and $\mathbf{k}$ is taken to be a diagonal tensor, $\operatorname{diag}\left(k_{x}, k_{z}\right)$. Combining Darcy's law with a statement of conservation of mass allows us to express the governing equations in terms of the so-called pressure and saturation equations:

$$
\begin{aligned}
& \nabla \cdot(\lambda(S) \mathbf{k} \cdot \nabla p)=q, \\
& \frac{\partial S}{\partial t}+\mathbf{v} \cdot \nabla f(S)=0,
\end{aligned}
$$

where $\lambda$ is the total mobility, $q$ is a source term, $f$ is the flux function of water, and $\mathbf{v}$ is the total velocity, which are respectively given by:

$$
\begin{gathered}
\lambda(S)=\frac{k_{r w}(S)}{\mu_{w}}+\frac{k_{r o}(S)}{\mu_{o}}, \\
f(S)=\frac{k_{r w}(S) / \mu_{w}}{k_{r w}(S) / \mu_{w}+k_{r o}(S) / \mu_{o}}, \\
\mathbf{v}=\mathbf{v}_{w}+\mathbf{v}_{o}=-\lambda(S) \mathbf{k} \cdot \nabla p .
\end{gathered}
$$


The above descriptions are referred to as the fine model of the two phase flow problem.

Next, we wish to develop a coarse scale description for two phase flow in heterogeneous porous media. Previous approaches for upscaling such systems are discussed by many authors; e.g., $[6,3,10,15]$. In most upscaling procedures, the coarse scale pressure equation is of the same form as the fine scale equation (2.2), but with an equivalent grid block permeability tensor $\mathbf{k}^{*}$ replacing $\mathbf{k}$. For a given coarse scale grid block, the tensor $\mathbf{k}^{*}$ is generally computed through the solution of the pressure equation over the local fine scale region corresponding to the particular coarse block [9]. Coarse grid $\mathbf{k}^{*}$ computed in this manner have been shown to provide accurate solutions to the coarse grid pressure equation. We note that some upscaling procedures additionally introduce a different coarse grid functionality for $\lambda$, though this does not appear to be essential in our formulation.

In this work, the proposed coarse model is upscaling the pressure equation (2.2) to obtain the velocity field on the coarse grid and use it in (2.3) to resolve the saturation on the coarse grid. A finite volume element method is implemented to upscale the pressure equation (2.2). Finite volume is chosen, because, by its construction, it enjoys the numerical local conservation which is important in groundwater and reservoir simulations. We note that similar procedure for this pressure equation upscaling has been implemented in [25]. First, we describe briefly several geometrical terminologies related to the method. Let $\mathcal{K}^{h}$ denote the collection of coarse elements/rectangles $K$, whose side lengths in $x$ - and $z$-direction, respectively, are $h_{x}$ and $h_{z}$, and the maximum of those two is $h$. We describe the construction of the control volumes as follows. Consider a coarse element $K$, and let $\xi_{K}$ be its center. The element $K$ is divided into four rectangles of equal area by connecting $\xi_{K}$ to the midpoints of the element's edges. We denote these quadrilaterals by $K_{\xi}$, where $\xi \in Z_{h}(K)$ are the vertices of $K$. Also, we denote by $Z_{h}=\bigcup_{K} Z_{h}(K)$ the collection of all vertices and by $Z_{h}^{0} \subset Z_{h}$ the vertices which do not lie on the Dirichlet boundary of $\Omega$. The control volume $V_{\xi}$ is defined as the union of the quadrilaterals $K_{\xi}$ sharing the vertex $\xi$.

The key idea of the method is the construction of base functions on the coarse grids such that these base functions capture the small scale information on each of these coarse grids. The method that we use follows its finite element counterpart presented in [23]. The base functions are constructed from the solution of the 
leading order homogeneous elliptic equation on each coarse element with some specified boundary conditions. Thus, if we consider a coarse element $K$ that has $d$ vertices, the local base functions $\phi^{i}, i=1, \cdots, d$ are set to satisfy the following elliptic problem:

$$
\begin{aligned}
-\nabla \cdot\left(\mathbf{k} \cdot \nabla \phi^{i}\right) & =0 & & \text { in } K \\
\phi^{i} & =g^{i} & & \text { on } \partial K,
\end{aligned}
$$

for some function $g^{i}$ defined on the boundary of the coarse element $K$. Hou et al. [23] have demonstrated that a careful choice of boundary condition would guarantee the performance of the base functions to incorporate the local information and, hence, improve the accuracy of the method. In this paper, the function $g^{i}$ for each $i$ varies linearly along $\partial K$. Thus, for example, in case of a constant diagonal tensor, the solution of (2.7) would be a standard linear/bilinear base function. We note that as usual we require $\phi^{i}\left(\xi_{j}\right)=\delta_{i j}$. Finally, a nodal base function associated with the vertex $\xi$ in the domain $\Omega$ are constructed from the combination of the local base functions that share this $\xi$ and zero elsewhere. These nodal base functions are denoted by $\left\{\psi_{\xi}\right\}_{\xi \in Z_{h}^{0}}$.

Having described the base functions, we denote by $V^{h}$ the space of our approximate pressure solution which is spanned by the base functions $\left\{\psi_{\xi}\right\}_{\xi \in Z_{h}^{0}}$. Now, we may formulate the finite dimensional problem corresponding to finite volume element formulation of (2.2). A statement of mass conservation on a control volume $V_{\xi}$ is formed from (2.2), where now the approximate solution is written as a linear combination of the base functions. Assembly of this conservation statement for all control volumes would give the corresponding linear system of equations that can be solved accordingly. It is obvious that the number of the control volumes $V_{\xi}$ has to be equal to the dimension of the space $V^{h}$. The resulting linear system has incorporated the fine scale information through the involvement of the nodal base functions on the approximate solution. To be specific, the problem now is to seek $p^{h} \in V^{h}$ with $p^{h}=\sum_{\xi \in Z_{h}^{0}} p_{\xi} \psi_{\xi}$ such that

$$
\int_{\partial V_{\xi}} \lambda(S) \mathbf{k} \cdot \nabla p^{h} \cdot \vec{n} d l=\int_{V_{\xi}} q d A,
$$

for every control volume $V_{\xi} \subset \Omega$. Here $\vec{n}$ defines the normal vector on the boundary of the control volume, $\partial V_{\xi}$ and $S$ is the fine scale saturation field. 
We note that concerning the base functions, a vertex-centered finite volume difference is used to solve (2.7) along with a harmonic average to approximate the permeability $\mathbf{k}$ at the edges of fine control volumes.

As mentioned earlier, the pressure solution may then be used to compute the total velocity field at the coarse scale level, denoted by $\overline{\mathbf{v}}=\left(\bar{v}_{x}, \bar{v}_{z}\right)$ via (2.6). In general, the following equations are used to compute the velocities in horizontal and vertical directions, respectively:

$$
\begin{aligned}
& \bar{v}_{x}=-\frac{1}{h_{z}} \sum_{\xi \in Z_{h}^{0}} p_{\xi}\left(\int_{E} \lambda(S) k_{x} \frac{\partial \psi_{\xi}}{\partial x} d z\right), \\
& \bar{v}_{z}=-\frac{1}{h_{x}} \sum_{\xi \in Z_{h}^{0}} p_{\xi}\left(\int_{E} \lambda(S) k_{z} \frac{\partial \psi_{\xi}}{\partial z} d x\right),
\end{aligned}
$$

where $E$ is the edge of $V_{\xi}$. Furthermore, for the control volumes $V_{\xi}$ adjacent to Dirichlet boundary (which are half control volumes), we can derive the velocity approximation using the conservation statement derived from (2.2) on $V_{\xi}$. One of the terms involved is the integration along part of Dirichlet boundary, while the rest of the three terms are known from the adjacent internal control volumes calculations. The integration of forcing function may be approximated by midpoint rule. This way, we have the following equations $(l, b, r$, and $t$ stand for left, bottom, right, and top, respectively):

$$
\begin{aligned}
\bar{v}_{x}^{l}= & \bar{v}_{x}^{r}+0.5 h_{x} / h_{z}\left(\bar{v}_{z}^{t}-\bar{v}_{z}^{b}\right)-0.5 h_{x} \bar{q} \\
& \quad \text { or left Dirichlet boundary, } \\
\bar{v}_{z}^{b}= & \bar{v}_{z}^{t}+0.5 h_{z} / h_{x}\left(\bar{v}_{x}^{r}-\bar{v}_{x}^{l}\right)-0.5 h_{z} \bar{q}
\end{aligned}
$$

for bottom Dirichlet boundary.

The right and the top Dirichlet boundary conditions are defined similarly. It has been well known that these approximations give a second order accuracy to the velocity computation. We have analyzed both numerically and analytically our two-scale finite volume methods and the results will be published elsewhere [22].

In this paper we will consider two different coarse models for the saturation equation. One of them is a simple/primitive model where we use only the coarse 
scale velocity to update the saturation field on the coarse grid, i.e.,

$$
\frac{\partial \bar{S}}{\partial t}+\overline{\mathbf{v}} \cdot \nabla f(\bar{S})=0
$$

In this case no upscaling of the saturation equation is performed. This kind of technique in conjunction with the upscaling of absolute permeability is commonly used in applications (e.g., $[12,11,10])$. The difference of our approach is that the coupling of the small scales is performed through the finite volume element formulation of the global problem and the small scale information of the velocity field can be easily recovered. Within this upscaling framework, we use $\bar{S}$ instead of $S$ in (2.8). If the saturation profile is smooth, this approximation is of first order. In the coarse blocks where the discontinuities of $S$ are present, we need to modify the stiffness matrix corresponding to these blocks. The latter requires the values of the fine scale saturation. In our computation we will not do this. We simply use $\lambda(\bar{S})$ in (2.8).

In addition to the above described coarse model, we will also revisit a coarse model on the saturation proposed by [17], which uses $\lambda(S)=1$ and $f(S)=S$. This model was derived using perturbation argument for (2.3), in which the saturation, $S$, and the velocity, $\mathbf{v}$, on the fine scale are assumed to be the sum of their volume-averaged and fluctuating components,

$$
\mathbf{v}=\overline{\mathbf{v}}+\mathbf{v}^{\prime}, \quad S=\bar{S}+S^{\prime}
$$

Here, the overbar quantities designate the volume average of fine scale quantities over coarse blocks. For simplicity we will assume that the coarse blocks are rectangular, which allows us to state that (cf. [33])

$$
\overline{\nabla f}=\nabla \bar{f} .
$$

Substituting (2.13) into the saturation equation for single phase and averaging over coarse blocks, we obtain

$$
\frac{\partial \bar{S}}{\partial t}+\overline{\mathbf{v}} \cdot \nabla \bar{S}+\overline{\mathbf{v}^{\prime} \cdot \nabla S^{\prime}}=0
$$

The term $\overline{\mathbf{v}^{\prime} \cdot \nabla S^{\prime}}$ represents subgrid effects due to the heterogeneities of convection. This term can be modeled using the equation for $S^{\prime}$ that is derived by 
subtracting (2.14) from the fine scale equation (2.3)

$$
\frac{\partial S^{\prime}}{\partial t}+\overline{\mathbf{v}} \cdot \nabla S^{\prime}+\mathbf{v}^{\prime} \cdot \nabla \bar{S}+\mathbf{v}^{\prime} \cdot \nabla S^{\prime}=\overline{\mathbf{v}^{\prime} \cdot \nabla S^{\prime}} .
$$

This equation can be solved along the characteristics $d \mathbf{x} / d t=\overline{\mathbf{v}}$ by neglecting higher order terms. Carrying out the calculations in an analogous manner to the ones performed in [17], we can easily obtain the following coarse scale saturation equation:

$$
\frac{\partial \bar{S}}{\partial t}+\overline{\mathbf{v}} \cdot \nabla \bar{S}=\nabla \cdot \mathbf{D}(\mathbf{x}, t) \nabla \bar{S}(\mathbf{x}, t)
$$

where $\mathbf{D}(\mathbf{x}, t)$ is the macro-diffusive tensor, whose entries are written as

$$
D_{i j}(\mathbf{x}, t)=\left[\int_{0}^{t} \overline{v_{i}^{\prime}(\mathbf{x}) v_{j}^{\prime}(\mathbf{x}(\tau))} d \tau\right] .
$$

Next, it can be easily shown that the coefficient of diffusion can be approximated up to the first order by

$$
D_{i j}(\mathbf{x}, t)=\overline{v_{i}^{\prime}(\mathbf{x}) L_{j}},
$$

where $L_{j}$ is the displacement of the particle in $j$ direction that starts at point $\mathbf{x}$ and travels with velocity $\mathbf{- v}$. The diffusion term in the coarse model for the saturation field (2.15) represents the effects of the small scales on the large ones. Note that the diffusion coefficient is a correlation between the velocity perturbation and the displacement. This is different from [17], where the diffusion is taken to be proportional to the length of the coarse scale trajectory. Using our upscaling methodology for the pressure equation, we can recover the small scale features of the velocity field that allows us to compute the fine scale displacement.

For the nonlinear flux, $f(S)$, we can use a similar argument by expanding $f(S)=f(\bar{S})+f_{S}(\bar{S}) S^{\prime}+\ldots$ In this expansion we will take into account only linear terms and assume that the flux is nearly linear. This case is similar to the linear case, and the analysis can be carried out in an analogous manner. The resulting coarse scale equation has the form

$$
\frac{\partial \bar{S}}{\partial t}+\overline{\mathbf{v}} \cdot \nabla \bar{S}=\nabla \cdot f_{S}(\bar{S})^{2} \mathbf{D}(\mathbf{x}, t) \nabla \bar{S}(\mathbf{x}, t),
$$


where $\mathbf{D}(\mathbf{x}, t)$ is the macro-diffusive tensor corresponding to the linear flow. This formulation has been derived within stochastic framework in [26]. We note that the higher order terms in the expansion of $f(S)$ may result in other effects that have not been studied extensively to the best of our knowledge. In [16] the authors use a similar formulation, though their implementation is different from ours. A couple of numerical examples for nonlinear flux $f(S)$ with $\lambda(S)=1$ will be presented.

\section{Numerical results}

We now present numerical results that demonstrate the accuracy and limitation of our model compared to the fine scale model. As in [17], the systems considered are representative of cross sections in the subsurface. We therefore set the system length in the horizontal direction $x\left(L_{x}\right)$ to be greater than the formation thickness $\left(L_{z}\right)$; in the results presented below, $L_{x} / L_{z}=5$. The fine model uses $120 \times 120$ rectangular elements. The absolute permeability is set to be $\operatorname{diag}(k, k)$. Thus, the fine grid permeability fields are $121 \times 121$ realizations of prescribed overall variance (quantified via $\sigma^{2}$, the variance of $\log k$ ), correlation structure, and covariance model. We consider models generated using GSLIB algorithms [8], characterized by spherical and exponential variograms [30, 8]. The dimension of the coarse models range from $10 \times 10$ to $40 \times 40$ elements and are generated using a uniform coarsening of the fine grid description.

For the spherical and exponential variogram models, the dimensionless correlation lengths (nondimensionalized by $L_{x}$ and $L_{z}$, respectively) are designated by $l_{x}$ and $l_{z}$. As discussed in [17], because our dispersivity model is pre-asymptotic, we do not expect it to be applicable to the case of very small $l_{x}$. Therefore, in the results below, we restrict ourselves to $l_{x} \geq 0.1$.

We set the relative permeabilities of oil and water to be simple quadratic functions of their respective saturations; i.e., $k_{r w}=S^{2}$ and $k_{r o}=(1-S)^{2}$, where $S$ is the water saturation. Specifically for the first two examples, where we deal with pressure dependence on the saturation, we set our viscosity ratio $\mu_{o} / \mu_{w}=5$. In all cases we fix pressure and saturation $(S=1)$ at the inlet edge of the model $(x=0)$ and also fix pressure at the outlet $\left(x=L_{x}\right)$. The top and bottom boundaries are closed to flow. Results are presented in terms of the fraction of oil in the 
produced fluid (denoted by $F$, where $F=q_{o} / q$, with $q_{o}$ being the volumetric flow rate of oil produced at the outlet edge and $q$ the volumetric flow rate of total fluid produced at the outlet edge) versus pore volumes injected (PVI). PVI is analogous to dimensionless time and is defined as $q t / V_{p}$, where $t$ is dimensional time and $V_{p}$ is the total pore volume of the system. In this study, we applied our models to a variety of permeability fields.

Our first example in Figure 1 is for the case $l_{x}=0.4, l_{z}=0.04$, and $\sigma=1.5$. An exponential variogram is used to generate the permeability realization. In the following two figures, the $120 \times 120$ fine model is represented by solid lines, while the coarse models are represented by the dashed lines and dotted lines, depending on the coarse model's dimension. On the top plot, the coarse model were run on $10 \times 10$ elements (dotted lines) and $30 \times 30$ elements (dashed lines). On the bottom plot, the coarse model were run on $20 \times 20$ elements (dotted lines) and $40 \times 40$ elements (dashed lines). In both of these plots, the coarse model overpredicts the breakthrough time and continues to overpredict the production of the displaced fluid until PVI $\approx 1$. After that time the comparison shows that the coarse model agrees reasonably well with the fine model. Also, it can be observed that the larger coarse models are more accurate in general. For example, the $40 \times 40$ coarse scale model gives a reasonable approximation of the fine scale model.

For the second example, we consider an isotropic field. Figure 2 shows comparison of the fractional flow for case $l_{x}=0.1, l_{z}=0.1$, and $\sigma=1.0$. Both plots in this figure show a good agreement between the fine model and coarse model, regardless of the coarse model dimensions. In conclusion, we would like to note that our coarse scale model tends to perform better for smaller correlation length. In particular, for the upscaling of high correlation length cases, we need larger coarse scale models. This difficulty can be relieved by introducing the nonuniform coarsening, which is a subject of further research.

Another important aspect that requires consideration is the ability of the coarse model to predict the saturation contour. In the following, we compare the saturation contours obtained from fine and coarse models with the same two permeability field scenarios as in the previous figures. The saturation contours are compared in the following fashion: the fine scale model result is averaged onto the coarse grid and then is overlapped with the result from the coarse model of 

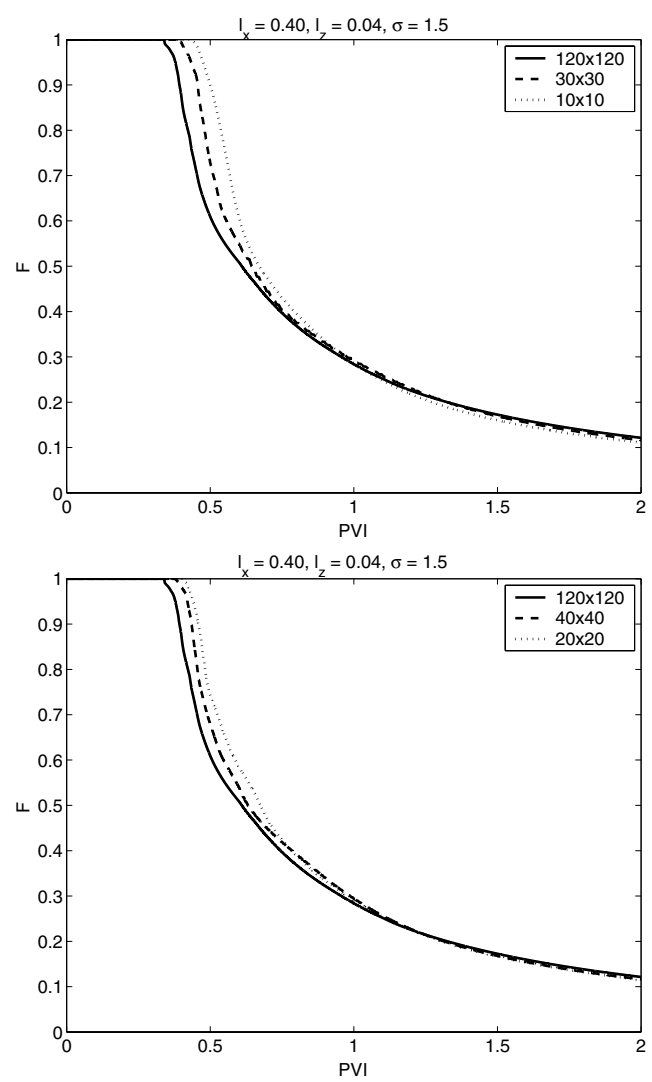

Figure 1 - Comparison of fractional flow of displaced fluid at the production edge for the case $l_{x}=0.4, l_{z}=0.04$, and $\sigma=1.5$ with exponential variogram, and $\mu_{o} / \mu_{w}=5$. Plots on the top are coarse model with $10 \times 10$ and $30 \times 30$ elements; plots on the bottom are coarse model with $20 \times 20$ and $40 \times 40$ elements.

$20 \times 20$ elements. In the subsequent figures, the following description is used: the upper plot shows $\bar{S}=0.10$, the middle plot shows $\bar{S}=0.30$, and the lower plot shows $\bar{S}=0.50$.

Figure 3 gives comparison of saturation contours at PVI $=0.15$, which is before breakthrough time. In general, the coarse model is able to predict the trends exhibited by the fine model, although for smaller values of saturation, it cannot quite follow the fingering indicated by the fine model as evident in upper and middle plots. For a higher value of saturation, however, the coarse 

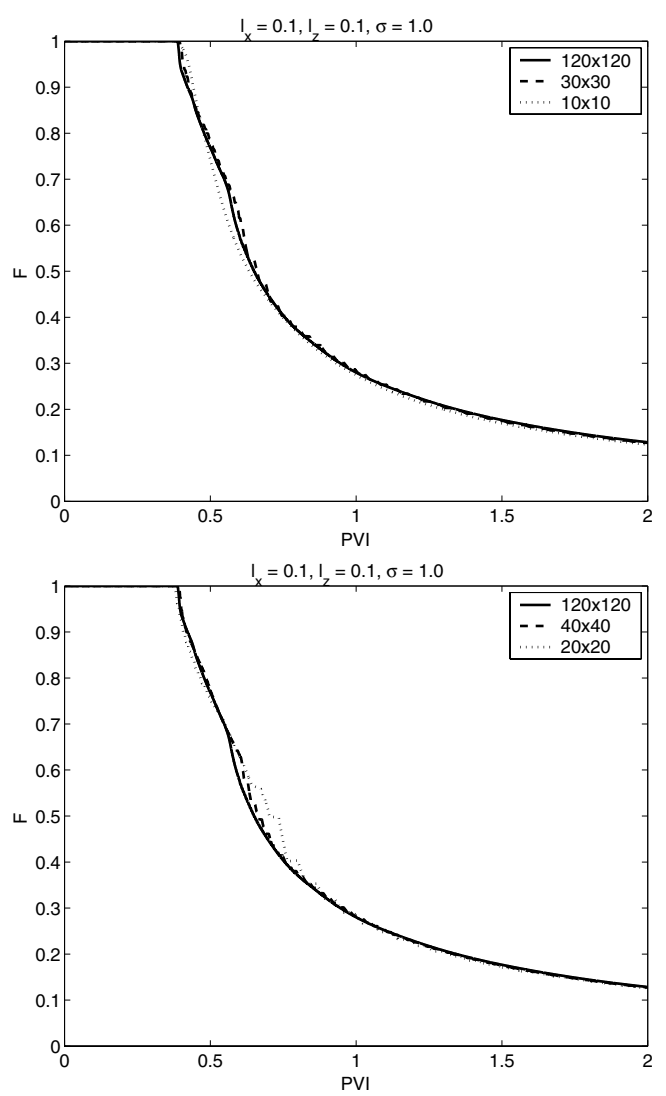

Figure 2 - Comparison of fractional flow of displaced fluid at the production edge for the case $l_{x}=0.1, l_{z}=0.1$, and $\sigma=1.0$ with spherical variogram, and $\mu_{o} / \mu_{w}=5$. Plots on the top are coarse model with $10 \times 10$ and $30 \times 30$ elements; plots on the bottom are coarse model with $20 \times 20$ and $40 \times 40$ elements.

model can follow the fingering indicated by the fine model as seen in lower plot. Similar behavior is shown in Figure 4 for isotropic field with $l_{x}=0.1, l_{z}=0.1$, and $\sigma=1$. These comparisons also show that the coarse model predicts the contour of saturation better for lower correlation lengths compared to the case with higher correlation length along the main flow direction, $l_{x}=0.4, l_{z}=0.04$, and $\sigma=1.5$.

At this stage, we present several numerical results of our coarse model with the macro-diffusion as described in Section 2. Comparison is made between 

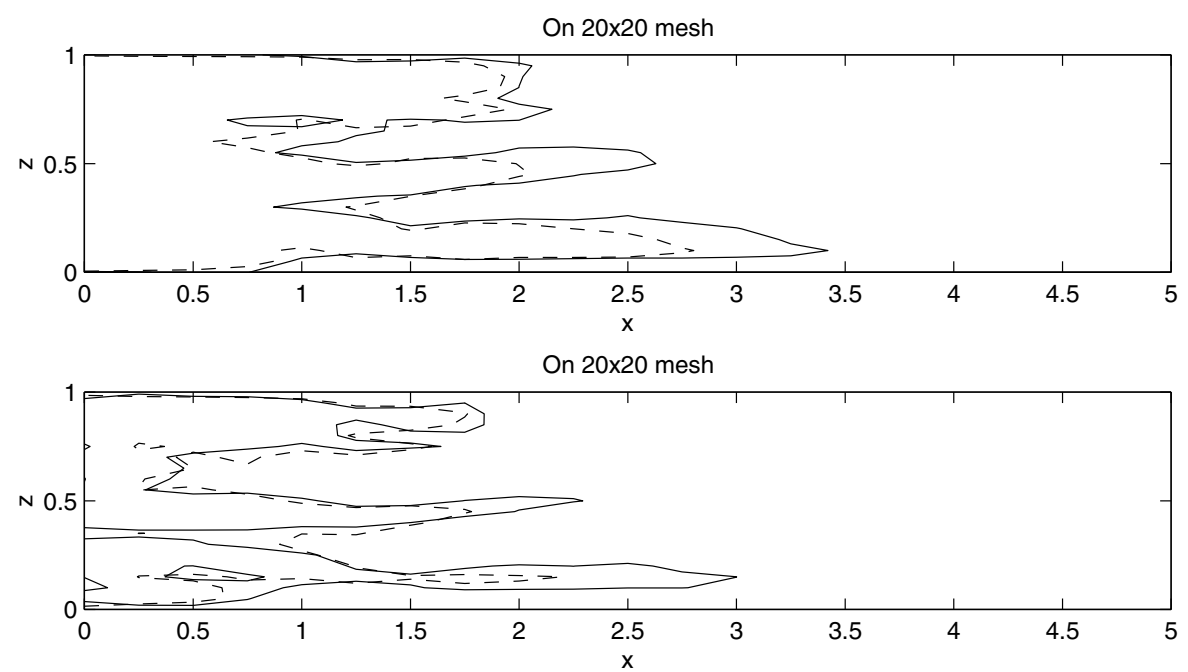

On 20x20 mesh

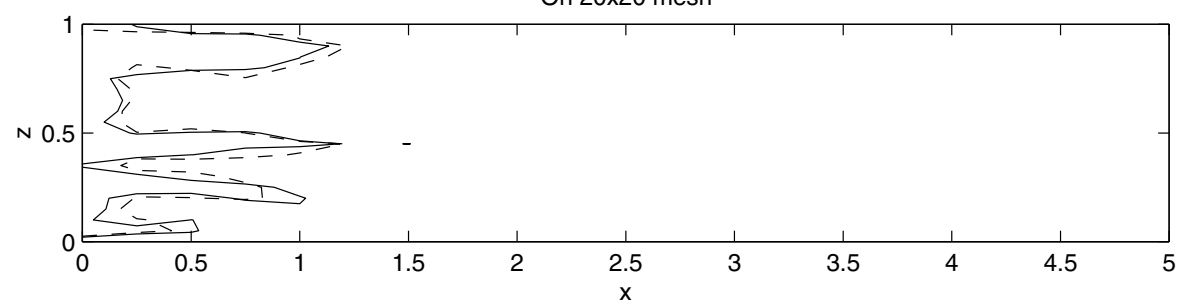

Figure 3 - Comparison of saturation contours at PVI $=0.15$ for the case $l_{x}=0.4$, $l_{z}=0.04$, and $\sigma=1.5$ with exponential variogram, and $\mu_{o} / \mu_{w}=5$. The solid lines represent the fine grid saturation after averaging onto the coarse grid, while the dashed lines represent the coarse model with $20 \times 20$ elements. Upper plots are the contour of $\bar{S}=0.10$, middle plots are the contour of $\bar{S}=0.30$, and lower plots are the contour of $\bar{S}=0.50$.

this transport coarse model with the primitive model, cf. (2.12). As opposed to the coarse model with macro-diffusion, by its nature, the primitive model does not account for the subgrid effects on the coarse grid. The macro-diffusion is computed using the approximation of the fine scale velocity field by sampling the base functions.

The performance of this macro-diffusion model is exhibited in Figures 5 and 6. The following notation and terminology are used in those two figures. The solid line represents the fine model run on $120 \times 120$ elements, which as before, serves 

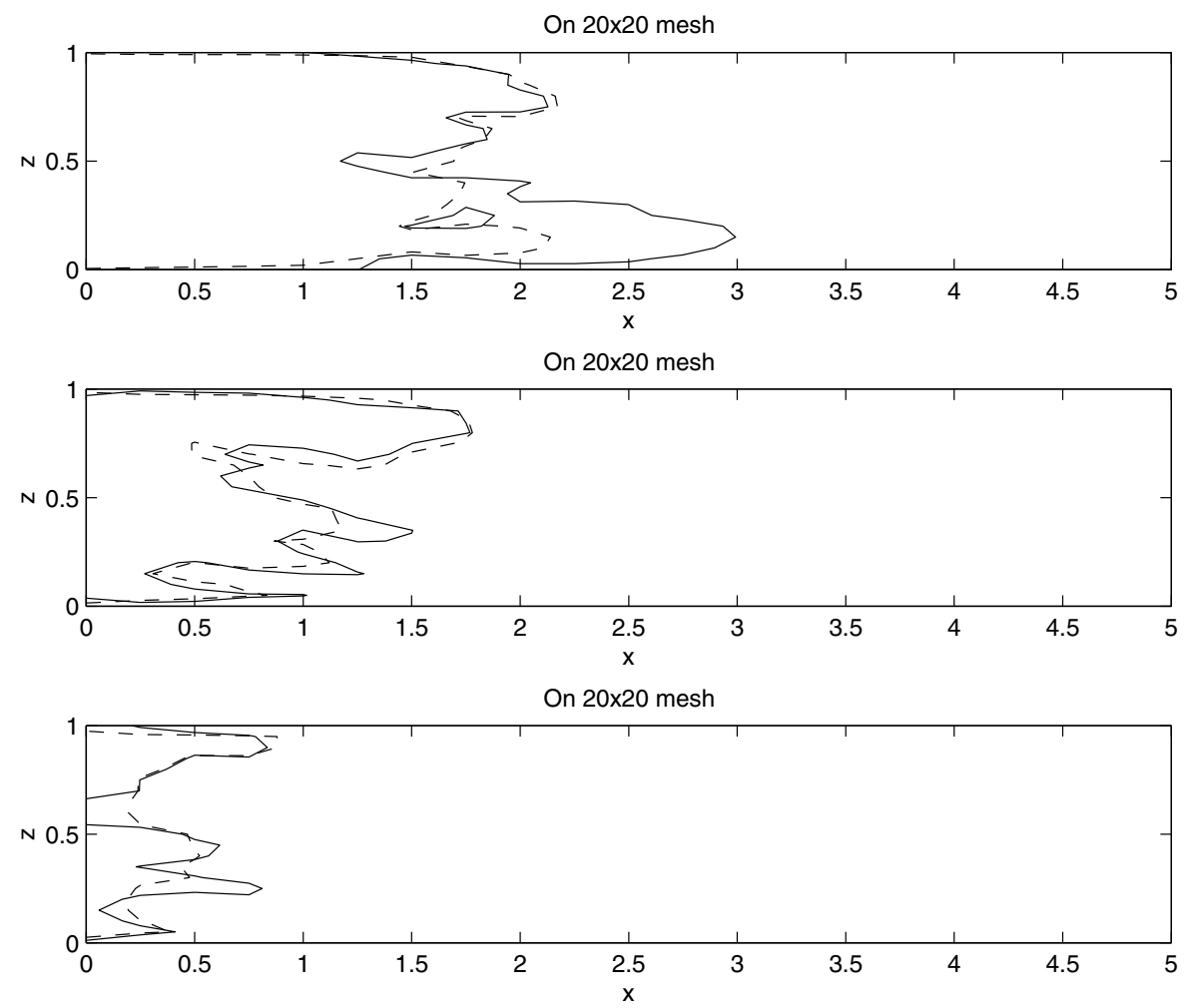

Figure 4 - Comparison of saturation contours at PVI $=0.15$ for the case $l_{x}=0.1$, $l_{z}=0.1$, and $\sigma=1.0$ with spherical variogram, and $\mu_{o} / \mu_{w}=5$. The solid lines represent the fine grid saturation after averaging onto the coarse grid, while the dashed lines represent the coarse model with $20 \times 20$ elements. Upper plots are the contour of $\bar{S}=0.10$, middle plots are the contour of $\bar{S}=0.30$, and lower plots are the contour of $\bar{S}=0.50$.

as a reference solution. The dashed line represents the primitive coarse model $(D=0)$, while the dotted line represents the coarse model with macro-diffusion (with D). All coarse models are run on the $10 \times 10$ elements.

Figure 5 shows the macro-diffusion model performance in the case of a linear flux function, $f(S)=S$ and $\lambda(S)=1$. The plot on the top corresponds to the isotropic permeability field with $l_{x}=0.1, l_{z}=0.1$, and $\sigma=1.5$, and the plot on the bottom corresponds to permeability field with $l_{x}=0.40, l_{z}=0.04$, and $\sigma=$ 1.5. For the isotropic case (top plot), it is evident from this figure that although 

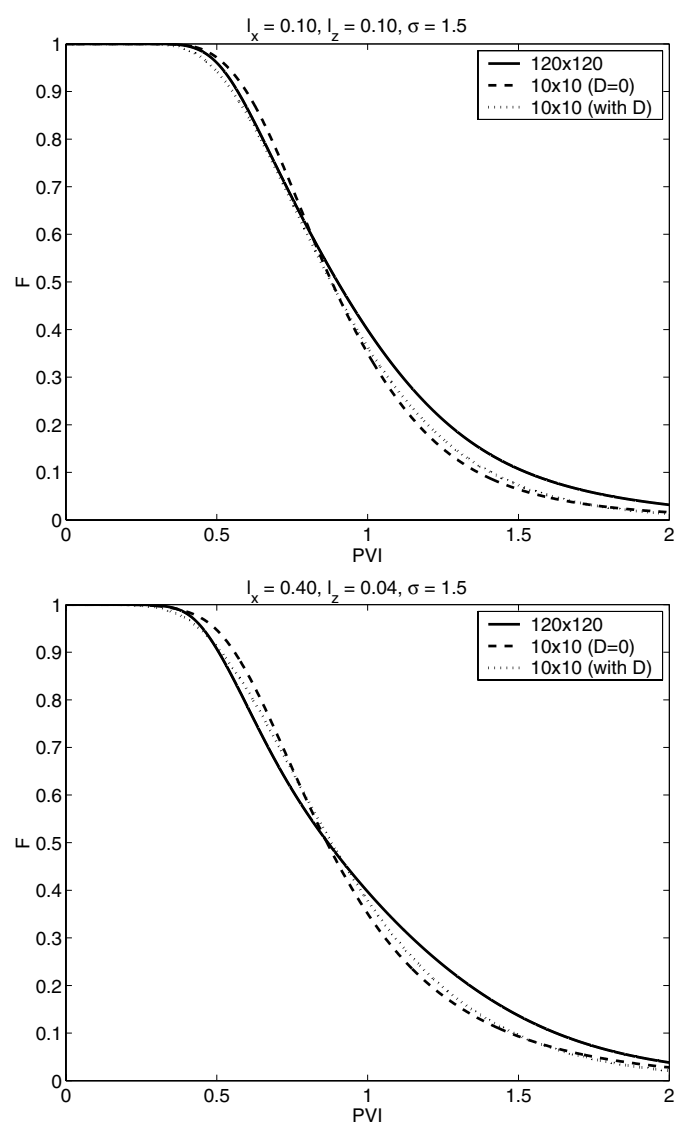

Figure 5 - Comparison of fractional flow of displaced fluid at the production edge. The flux function used is linear, $f(S)=S$. All coarse models are run on $10 \times 10$ elements. Plot on the top corresponds to $l_{x}=0.1, l_{z}=0.1$, and $\sigma=1.5$ with spherical variogram. Plot on the bottom corresponds to $l_{x}=0.40, l_{z}=0.04$, and $\sigma=1.5$ with spherical variogram.

the performance of the primitive coarse model seems to agree reasonably well with the fine model (specifically on the breakthrough time), the coarse model with macro-diffusion does improve the overall prediction. Conversely, when the correlation length is larger along the main flow direction (bottom plot), where now the diffusion caused by heterogeneity is stronger, the coarse model with macro-diffusion gives a better prediction compared to the primitive model. 
The performance of the coarse model with macro-diffusion in the case of nonlinear flux function is shown in Figure 6. Here we have used

$$
f(S)=\frac{5 S^{2}}{5 S^{2}+(1-S)^{2}} \quad \text { and } \quad \lambda(S)=1 .
$$

Again, the plot on the top corresponds to isotropic permeability field with $l_{x}=0.1, l_{z}=0.1$, and $\sigma=1.5$, and the plot on the bottom corresponds to permeability field with $l_{x}=0.40, l_{z}=0.04$, and $\sigma=1.5$. The significance of the macro-diffusion model in these two plots are obvious, in that the macrodiffusion model circumvents the primitive model in predicting the production on and shortly after the breakthrough. Also in this nonlinear flux function case, the model does not seem to be sensitive to the prescribed correlation structures.

To summarize, these computations reveal that the macro-diffusion resulting from the heterogeneity in the flow affects the coarse grid model, which may not be easily disregarded. Moreover, although solely based on the first order approximation, our proposed macro-diffusion model gives a reasonably well performance compared to the widely used primitive model.

Finally, we note that the viscous coupling is not taken into account in the macrodispersion model. In $[2,21]$ the authors investigated the viscous coupling and their findings indicate that the distinct dispersive regimes can occur depending on the relative strength of nonlinearity and heterogeneity. In particular, the viscosity ratio plays an important role in the stability of the fingering [2]. In the future we plan to use these results for developing new upscaling techniques for two-phase flow. For these approaches the upscaled mobility functions, $\lambda^{*}(\bar{S})$, that is different from $\lambda(\bar{S})$, will be employed. More general mobility functions, $\lambda^{*}(\bar{S}, \nabla \bar{S})$, that depends on both $\bar{S}$ and $\nabla \bar{S}$, will be also considered. We have employed the latter in a different upscaling framework in one of our previous works [14].

Another important class of flow in porous media problems is the unsaturated/saturated water flow governed by Richards' equation [4, 29]. This equation comes up from the simplification of the two phase water-air flow problem, where it is assumed that the temporal variation of the water saturation/water content is significantly larger than the temporal variation of the water pressure, and that the air phase is infinitely mobile so that the air pressure remains constant in 

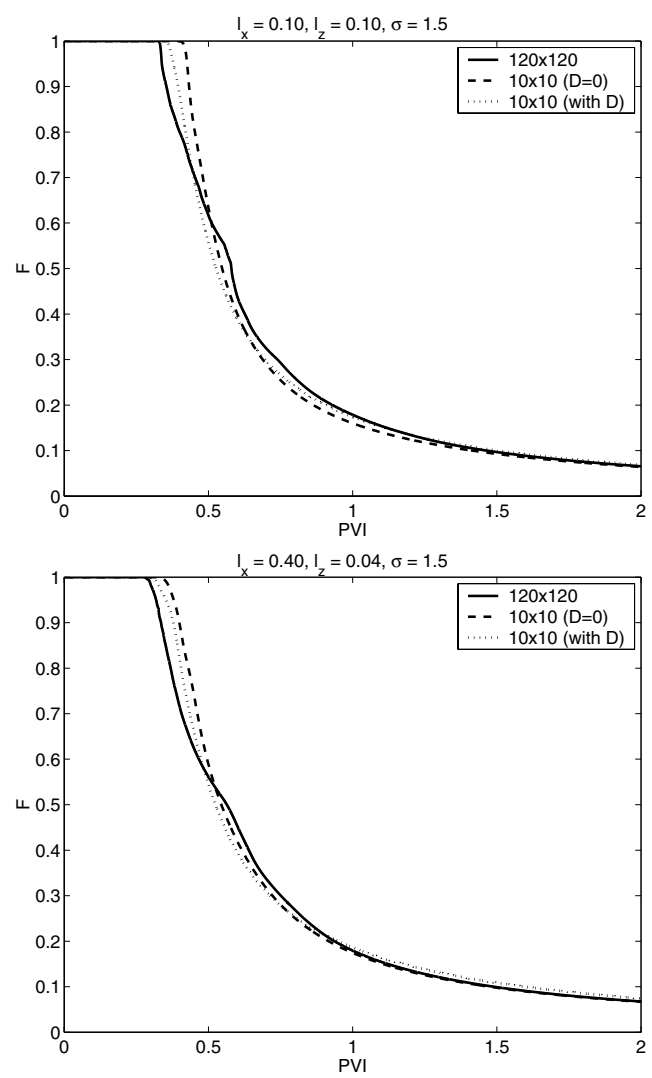

Figure 6-Comparison of fractional flow of displaced fluid at the production edge. The flux function used is nonlinear, $f(S)=\frac{5 S^{2}}{5 S^{2}+(1-S)^{2}}$. All coarse models are run on $10 \times 10$ elements. Plot on the top corresponds to $l_{x}=0.1, l_{z}=0.1$, and $\sigma=1.5$ with spherical variogram. Plot on the bottom corresponds to $l_{x}=0.40, l_{z}=0.04$, and $\sigma=1.5$ with spherical variogram.

the atmospheric level. The typical Richards' equation that we consider here is the so-called mixed formulation, in which the mass storage and transport are expressed in terms of water content and pressure head, respectively:

$$
\frac{\partial \theta(p)}{\partial t}-\nabla \cdot\left(\mathbf{k} k_{r w}(p) \nabla p\right)-\frac{\partial\left(k_{z} k_{r w}(p)\right)}{\partial z}=0,
$$

where $p$ denotes the water pressure head, $\theta(p)$ denotes the water content that depends on the pressure head $p$. 

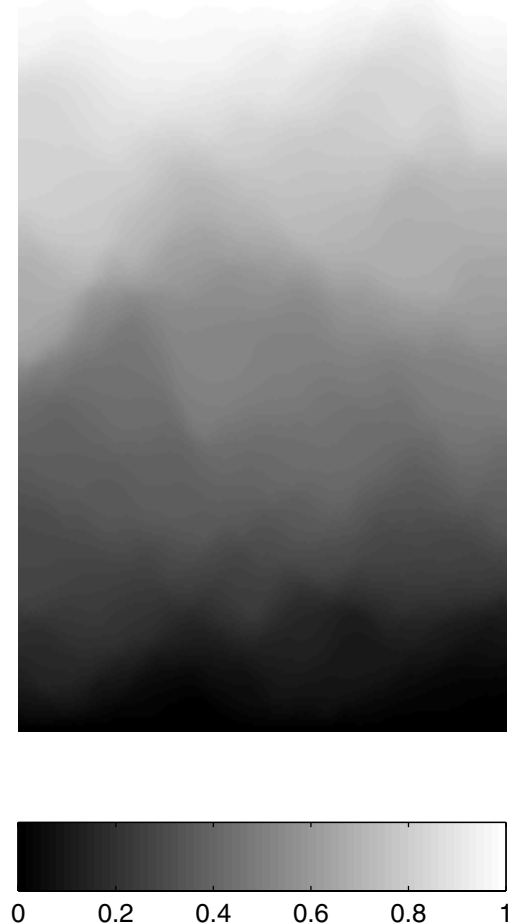
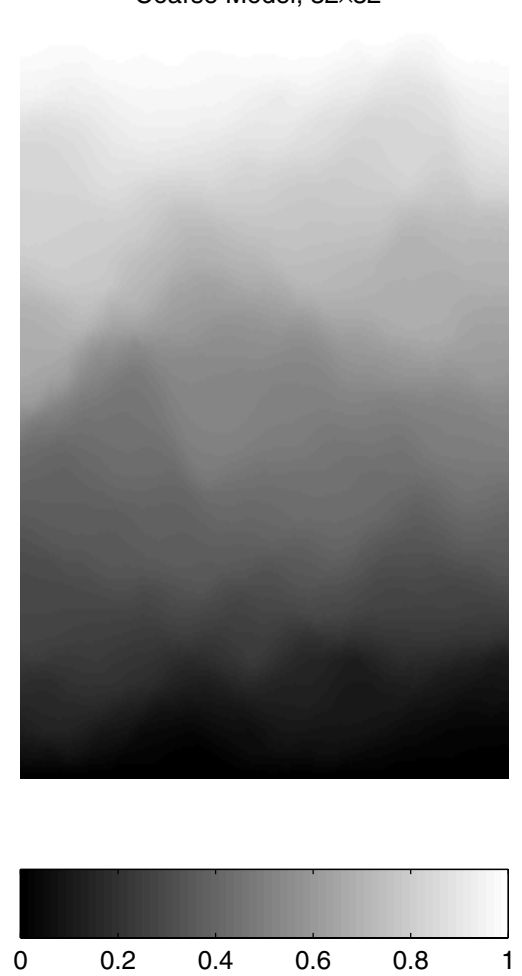

Figure 7 - Two-scale approximation of the Richards' equation. Comparison of water pressure between the fine model (left) and the coarse model (right).

The two-scale finite volume method described in Section 2 is applied to (3.18), where the resulting coarse model employs the same base functions as the linear problem. This approximation is motivated by the homogenization results of this class of equation [27, 28]. The analysis and more detailed description of this application will appear elsewhere.

One numerical example that we consider is a typical water infiltration shown in Figure 7. A fixed pressure is given on the top and bottom boundaries, and there is no flow on the lateral boundaries. A vertically linear initial pressure is imposed in the domain. The permeability field is generated with $l_{x}=0.1, l_{z}=0.1$, and $\sigma=1.5$. The water content and the relative permeability use exponential model, i.e., $\theta(p)=\exp (p)$ and $k_{r w}(p)=\exp (p)$. We note that the nonlinearity 
of Richards' equation is handled using the Picard iteration first proposed in [4]. The figure shows comparison of the water pressure plotted on $257 \times 257$ grids obtained using the fine model (left) and the coarse model (right). The fine model uses $256 \times 256$ elements, while the coarse model uses $32 \times 32$ elements. It is apparent from the figure that the coarse model agrees with the fine model.

\section{Summary}

In this paper we considered subgrid models for porous media flows. Upscaling procedures have been proposed for some multiphase flow applications and numerical results are presented. The numerical calculation of pressure and transport equations is accomplished in a consistent manner, providing a unified coarse scale model. The model was applied to a number of example cases involving heterogeneous permeability fields, varying linear and nonlinear fine scale flux functions. In essentially all cases considered, the subgrid model performed well on relatively coarse grids.

\section{Acknowledgement}

This work was partially supported by the NSF Grant EIA-0218229.

\section{REFERENCES}

[1] T. Arbogast and S.L. Bryant, Numerical subgrid upscaling for waterflood simulations. TICAM Report 01-23, http://www.ticam.utexas.edu/reports/2001/index.html.

[2] V. Artus and B. Noetinger, Macrodispersion approach for upscaling two-phase, immiscible flows in heterogeneous porous media. Presented at the 8th European Conference for the Mathematics of Oil Recovery, Freiberg, Germany, September (2002).

[3] J.W. Barker and S. Thibeau, A critical review of the use of pseudo-relative permeabilities for upscaling. SPE Res. Eng. 12 (1997), 138-143.

[4] M.A. Celia, E.T. Bouloutas and R.L. Zarba, A general mass-conservative numerical solution for the unsaturated flow equation. Water Resour. Res., (1990), pages 1483-1496.

[5] Z. Chen, R.E. Ewing and Z. Shi (editors), Numerical treatment of multiphase flows in porous media, volume 552 of Lecture Notes in Physics, Berlin, (2000). Springer-Verlag.

[6] M.A. Christie, Upscaling for reservoir simulation. J. Pet. Tech., (1996), pages 1004-1010.

[7] N.H. Darman, G.E. Pickup and K.S. Sorbie, A comparison of two-phase dynamic upscaling methods based on fluid potentials. Computational Geosciences, 6 (2002), 5-27. 
[8] C.V. Deutsch and A.G. Journel, GSLIB: Geostatistical software library and user's guide, 2nd edition. Oxford University Press, New York, (1998).

[9] L.J. Durlofsky, Numerical calculation of equivalent grid block permeability tensors for heterogeneous porous media. Water Resour. Res., 27 (1991), 699-708.

[10] L.J. Durlofsky, Coarse scale models of two phase flow in heterogeneous reservoirs: Volume averaged equations and their relationship to the existing upscaling techniques. Computational Geosciences, 2 (1998), 73-92.

[11] L.J. Durlofsky, R.A. Behrens, R.C. Jones and A. Bernath, Scale up of heterogeneous three dimensional reservoir descriptions. SPE paper 30709, (1996).

[12] L.J. Durlofsky, R.C. Jones and W.J. Milliken, A nonuniform coarsening approach for the scale up of displacement processes in heterogeneous media. Advances in Water Resources, 20 (1997), 335-347.

[13] W.E. and B. Engquist, The heterogeneous multi-scale methods. Comm. Math. Sci., 1 (1) (2003).

[14] Y. Efendiev and L. Durlofsky, Accurate subgrid models for two phase flow in heterogeneous reservoirs. Paper SPE 79680 presented at the SPE Reservoir Simulation Symposium, Houston, June 3-5, (2003).

[15]Y.R. Efendiev, Exact upscaling of transport in porous media and its applications. IMA Preprint Series, 1724, October, 2000, http://www.ima.umn.edu/preprints/oct2000/oct2000.html.

[16] Y.R. Efendiev and L.J. Durlofsky, Numerical modeling of subgrid heterogeneity in two phase flow simulations. Water Resour. Res., 38 (8) (2002).

[17] Y.R. Efendiev, L.J. Durlofsky and S.H. Lee, Modeling of subgrid effects in coarse scale simulations of transport in heterogeneous porous media. Water Resour. Res., 36 (2000), 20312041.

[18] Y.R. Efendiev, T.Y. Hou and X.H. Wu, Convergence of a nonconforming multiscale finite element method. SIAM J. Num. Anal., 37 (2000), 888-910.

[19] R.E. Ewing, Mathematical modeling and large-scale computing in energy and environmental research. In: The merging of disciplines: new directions in pure, applied and computational mathematics (Laramie, Wyo., 1985), pages 45-59. Springer, New York, (1986).

[20] R.E. Ewing, Upscaling of biological processes and multiphase flow in porous media. In: Fluid flow and transport in porous media: mathematical and numerical treatment (South Hadley, MA, 2001), volume 295 of Contemp. Math., pages 195-215. Amer. Math. Soc., Providence, RI, (2002).

[21] F. Furtado and F. Pereira, Crossover from nonlinearity controlled to heterogeneity controlled mixing in two-phase porous media flows. Computational Geosciences, 7 (2003), 115-135.

[22] V. Ginting, Analysis of a two-scale finite volume element for elliptic problem. Preprint, to be submitted. 
[23] T.Y. Hou and X.H. Wu, A multiscale finite element method for elliptic method for elliptic problems in composite materials and porous media. Journal of Computational Physics, 134 (1997), 169-189.

[24] T.Y. Hou, X.H. Wu and Z. Cai, Convergence of a multiscale finite element method for elliptic problems with rapidly oscillating coefficients. Math. Comp., 68 (227) (1999), 913-943.

[25] P. Jenny, S.H. Lee and H.A. Tchelepi, Multi-scale finite-volume method for elliptic problems in sub-surface flow simulation. Journal of Computational Physics, 187 (2003), 47-67.

[26] P. Langlo and M.S. Espedal, Macrodispersion for two-phase, immisible flow in porous media. Advances in Water Resources, 17 (1994), 297-316.

[27] A.K. Nandakumaran and M. Rajesh, Homogenization of a nonlinear degenerate parabolic differential equation. Electron. J. Differential Equations, 17 (2001), 1-19 (electronic).

[28] A. Pankov, G-convergence and homogenization of nonlinear partial differential operators. Kluwer Academic Publishers, Dordrecht, (1997).

[29] L.A. Richards, Capillary conduction of liquids through porous mediums. Physics, 1 (1931), 318-333.

[30] H. Wackernagle, Multivariate geostatistics: an introduction with applications. Springer, New York, (1998).

[31] T.C. Wallstrom, M.A. Christie, L.J. Durlofsky and D.H. Sharp, Effective flux boundary conditions for upscalling porous media equations. Transport in Porous Media, 46 (2002), 139-153.

[32] T.C. Wallstrom, S. Hou, M. Christie, L.J. Durlofsky, D.H. Sharp and Q. Zou, Application of effective flux boundary conditions to two-phase upscaling in porous media. Transport in Porous Media, 46 (2002), 155-178.

[33] W. Zijl and A. Trykozko, Numerical homogenization of two-phase flow in porous media. Comput. Geosci., 6 (1) (2002), 49-71. 